\title{
Towards an Approach for Multi-Device Interface Design
}

\author{
Rodrigo de Oliveira \\ UNICAMP - Campinas State University \\ PO Box 6176, 13084-971 Campinas, SP - Brazil \\ Phone: +55 (19) 3788-5838 \\ oliveira@ic.unicamp.br
}

\author{
Heloísa Vieira da Rocha \\ UNICAMP - Campinas State University \\ PO Box 6176, 13084-971 Campinas, SP - Brazil \\ Phone: +55 (19) 3788-5838 \\ heloisa@ic.unicamp.br
}

\begin{abstract}
This paper proposes an approach for multi-device interface design that extends pre-designed interfaces keeping the original application conceptual model without loosing its usability. The proposal reinterprets general aspects of well known HumanComputer Interaction (HCI) lifecycle models and can be extended to the design of any interactive product. When validated through a simple transformation between a large and a small screen interface in an HCI domain, it shall point to a much less maintenance cost/complexity and a better usability, ensuring the user's satisfaction.
\end{abstract}

\section{Categories and Subject Descriptors}

H.5.2 [Information Interfaces and Presentation]: User Interfaces - evaluation/methodology, graphical user interfaces (GUI), screen design, theory and methods, user-centered design.

\section{General Terms}

Design, Experimentation, Human Factors, Standardization.

\section{Keywords}

Adaptive web display, conceptual model, interface, mobile devices.

\section{INTRODUCTION}

Wireless interfaces for existing desktop applications have been truly encouraged due to the emerging mobile technologies. Some approaches suggest their design should start from the very beginning, focusing on each device's issues to get the most of them, like Avantgo (avantgo.com) and Usable Net (usablenet.com). Others try to make dynamic adaptations according to each device [4]. However, recent researches found that many adapted interfaces for the same application lack in usability [5], [6]. The assumptions reside on the fact that, despite linear transformations use appropriately the smaller screen space, they tend to make bigger changes on the interface resulting in confusion while re-finding/comparing information. Although isolated usability tests on these interfaces guarantee the desired goals, they can't do it when all the interfaces are put in use together. This leads to misconceptions, eventual user's flaws, distrust and, hence, bad usability.

The main problem here is that the original application conceptual model [9] is forgotten, giving space to a fresh and adapted one, which overlooks many of the user's cognitive processes, such as memory, learning and reasoning, among others.

This approach defends the hypothesis that one application shall not demand as much conceptual models as the number of final media devices to achieve its maximum usability. That is the only way to solve the user's decision making problem, allowing him to make the same and right choices to accomplish any task. To ensure the most important goal of Interaction Design is fulfilled, the approach demands similar usability indexes for the new interface, which can be achieved through usual design techniques, such as evaluations over all the design process. That is the result of a new design lifecycle model understanding, seen as unique and forever iterative.

\section{MULTI-DEVICE INTERFACE DESIGN APPROACH}

In order to avoid misconceptions about the term conceptual model, following is presented the definition given by [9] used in this work: conceptual model is a description of the proposed system in terms of a set of integrated ideas and concepts about what it should do, behave and look like, that will be understandable by the users in the manner intended. Stated so, there shouldn't be any misunderstandings with other interpretations, where it is seen in a much narrower perspective, corresponding to only the class diagram with conceptual classes, attributes and relationships between them [1], [10]. In fact, the definition used also concerns with the interface behavior (navigational model), look and feel (presentational model) and implementation.

\subsection{Sketching the Theory}

Let's consider a person that never had contact with whatever interactive product built to heat things. Now present him the stove with the task: "Heat this bottle of water the fast you can". As with anyone, the process of discovering how things work tends to be through inductive inference based reasoning. The user will draw conclusions about the objects or events on the basis of previous observations of similar objects or events. But he doesn't have any. 
So, the initial minutes will be certainly for exploration only. Eventually, the user will understand how the stove works and accomplish the task, improving his mental model with information like "the more I turn this knob to the right, the more this flame becomes warm", "the more this flame becomes warm, the faster the water heats", etc. Now present him a thermostat and another task analogous to the previous: "Heat this room the fast you can”. Both tasks are the same in their essence and so the user will certainly make his decision inductively, turning the thermostat knob most to the right. But it doesn't work the same way. Why is that?

The applications were both the same (to heat things) but their designers changed the conceptual model for their interfaces: they all were able to do similar things, they all had the same knob to turn but the behavior of the thermostat was different, always heating the room in a constant speed until the desired temperature is reached. There is no doubt the user could perfectly learn this new concept, but in a while, many usability attributes would be in risk, such as memorability, security, efficiency, etc. Figure 1 shows how Don Norman's interactive components can be overloaded when different conceptual models are allowed.

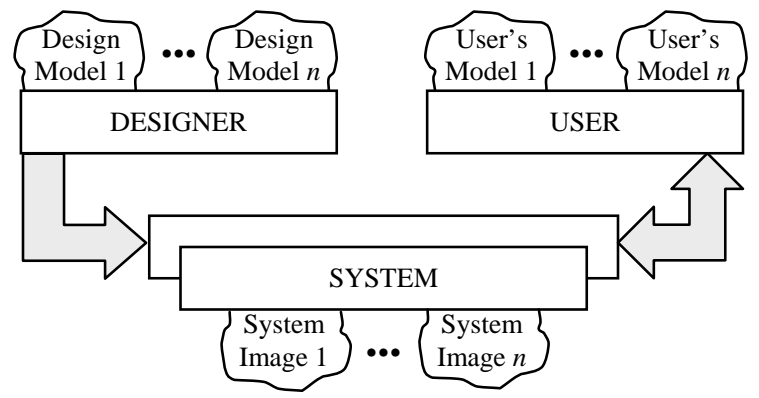

Figure 1. Interactive components with multiple conceptual models (adapted from [8]).

This approach suggests that it isn't just the interactive product to be evaluated, but also the application itself. This leads to a new understanding of well-know design lifecycle models.

\subsection{Reinterpreting the Lifecycle Models}

Many lifecycle models were proposed in HCI, like the star, the usability engineering and the interaction design models [9]. Although design is considered to be an iterative process in all of them, it has an ending point. As an example, the interaction design lifecycle model of [9] reaches the final product after one last evaluation which probably identifies that all the needs and usability goals were achieved. Supposing this product is an elearning environment for desktops, this means that, after some years of technological progress, when new user access needs will definitely appear calling for cellular and palmtop interfaces, another lifecycle will start. This new interface requirement is usually taken as such a big step to continue the old lifecycle. When that happens, the new interface generally brakes down the original conceptual model and the result will inevitably be considered by the user as a whole new application.

This approach proposes that no other lifecycle is needed because it is all about the same application. The final product turns to a new potential one over the old application and the entire process continues iteratively. Figure 2 presents this approach's lifecycle proposal.

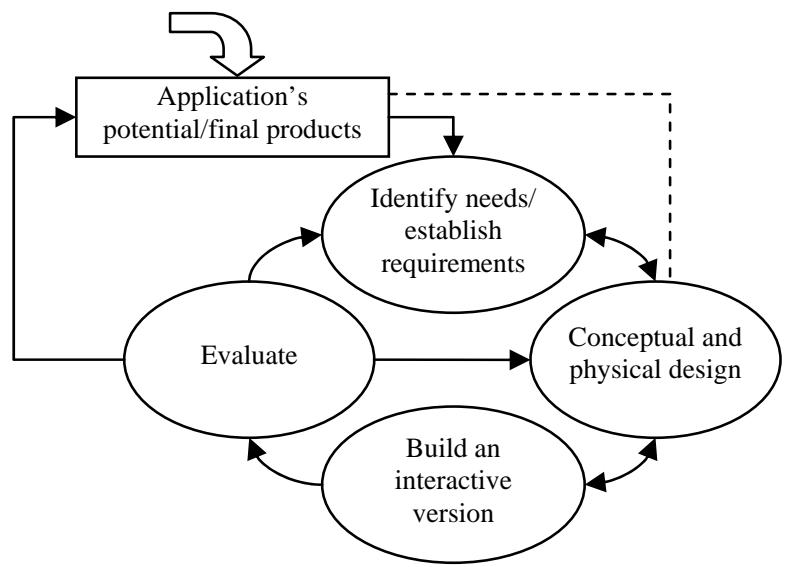

Figure 2. Approach's design lifecycle model (adapted from [9]).

According to Figure 2, the interaction design process has no end, starting with a potential product for some application and running forever iterative, even after the final product achievement. When that happens, designers concentrate on prospective user needs ensuring new potential related products will be identified and built according to the same design model. The dotted line on Figure 2 reinforces this dependency.

As a result of this interpretation for the lifecycle models, the interactive components relationship shown on Figure 3 is much closer to the original model [8], adding only the application's multi-interface nature.

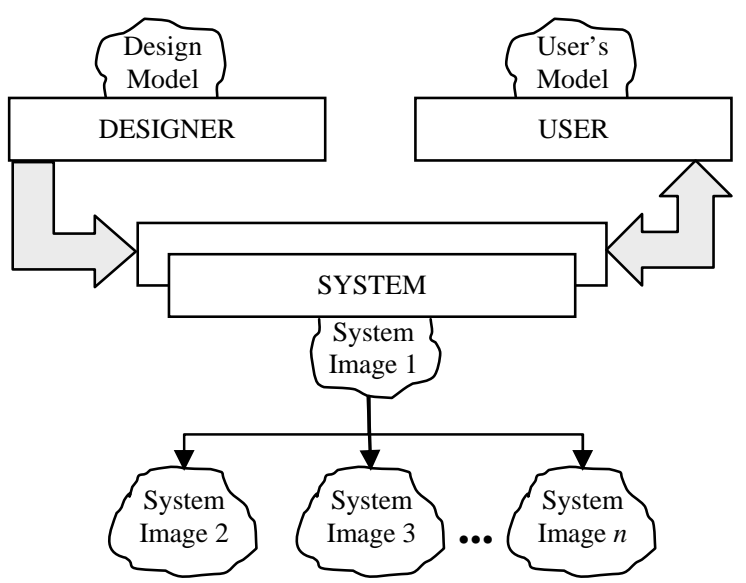

Figure 3. Interactive components with the same conceptual model (adapted from [8]).

\subsection{HCI Implementation Example}

Although this proposal scope has a much broader ambition for Interaction Design, the HCI field was chosen to validate this approach because of some good solutions proposed recently that can be mixed in this methodology molds to achieve its goals. There's no doubt the transition demanded from one conceptual model to $n$ physical designs can be a hard-work. As an example, the mobile interfaces needed for existent desktop applications 
face a great HCI challenge regarding the handhelds limited screen size. Following are listed three current approaches for solving this problem without changing the conceptual model:

- $\quad$ Smartview [7] - Thumbnail view of the original web page in zoom-out, partitioned in logical regions bounded with lines. After selected, the region is presented with good visibility inside the screen space (detailed view);

- Gateway [6] - Similar to Smartview, but dispenses the bounds and presents the detailed view over the thumbnail when selected;

- Summary Thumbnail [5] - The same previous thumbnail approach but with good content visibility via text summarization. The detailed view is not adapted, like in the direct migration.

In order to correctly apply the approach proposed in this paper, the application lifecycle model must continue its iteration process and each of these last approaches will be seen as resources to maintain the conceptual model with good usability. This means the final interface should use the Summary Thumbnail's visibility, the Smartview and/or Gateway's detailed view access and a better summarization technique, especially for navigational links to avoid some language misconceptions. Although Latent Semantic Analysis could overcome grammatical problems like synonymy and polysemy [3], the lexical analysis of [2] should be a better trade-off considering efficiency issues.

We plan to use this approach to achieve and evaluate the interface of Figure 4 for the TelEduc e-learning environment (http://teleduc.nied.unicamp.br/teleduc/). The validation will certainly be extended to many other web applications as the whole transformation process can be the same.

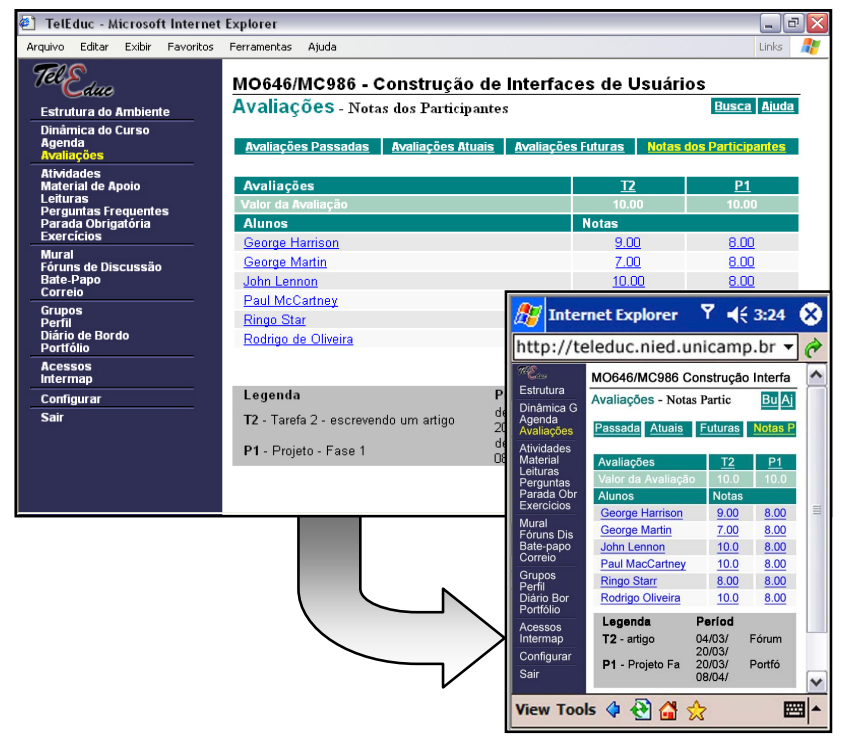

Figure 4. A design sketch for the TelEduc PDA interface obtained with this approach.

\section{CONCLUSIONS}

According to recent research findings, the proposed multi-device interface design approach elaborates a better framework for interaction design, with a particular contribution to the imminent mobile interfaces. It points to a new design perspective, not only for computational domains, but for any interdisciplinary field concerned with the design of interactive products. In particular, the HCI validation example may certainly help to diffuse many web applications around the mobile public.

\section{REFERENCES}

[1] Andrade, A. R.; Munson, E. V.; Pimentel, M. G. C. Engineering web applications with xml and xslt. In: Proceedings of LA-Web 2004, IEEE CS Press, Ribeirão Preto, São Paulo, pp. 86-93.

[2] Buyukkokten, O.; Kaljuvee, O; Garcia-Molina, H.; Paepcke, A.; Winograd, T. Efficient web browsing on handheld devices using page and form summarization. In: Proceedings of ACM Transactions on Information Systems (TOIS). New York, USA, v. 20, 2002. pp. 82-115.

[3] Furnas, G. W.; Deerwester, S.; Dumais, S. T.; Landauer T. K., Harshman, R. A.; Streeter, L. A.; Lochbaum, K. E. Information retrieval using a singular value decomposition model of latent semantic structure. In: Proceedings of 11th International Conference on Research \& Development in Information Retrieval, 1988, pp. 465-480.

[4] Hinz, M. Fiala, Z. Wehner, F. Personalization-based optimization of web interfaces for mobile devices. In: Proceedings of the Mobile HCI 2004, Glasgow, Scotland, pp. 204-215.

[5] Lam, H.; Baudisch, P. Summary thumbnails: readable overviews for small screen web browsers. In: Proceedings of CHI 2005, Portland, OR, pp. 681-690.

[6] Mackay, B.; Watters, C.; Duffy, J. Web page transformation when switching devices. In: Proceedings of the Mobile HCI 2004, Glasgow, Scotland, pp. 228-239.

[7] Milic-Frayling, N.; Sommerer, R. SmartView: enhanced document viewer for mobile devices. MSR Technical Report, 2002.

[8] Norman, D. The design of everyday things. Basic Books, New York, 1988.

[9] Preece, J.; Rogers, Y.; Sharp, H. Interaction design: beyond human-computer interaction. Wiley \& Sons, 2002.

[10] Rossi, G.; Schwabe, D.; Lyardet, F. Web application models are more than conceptual models. In: Proceedings of ER'99, Springer, Paris, France, 1999, pp. 239-252. 